\title{
СИСТЕМНЫЙ ПОДХОД К ВЫЯВЛЕНИЮ ДЕПРЕССИИ МЕТОДАМИ МАШИННОГО ОБУЧЕНИЯ
}

\section{SYSTEM APPROACH TO DETECTION OF DEPRESSION BY METHODS OF MACHINE LEARNING}

\section{E. Romanova}

Summary. A systematic approach to the study of the problem implies a systemic nature of the approach itself. This paper discusses options for constructing a classifier for recognizing the depressive states of members of society using machine learning methods. A method is proposed for constructing a classifier based on data from monitoring physical activity and characteristics of a lifestyle.

Keywords: data analysis, systems approach, classification, depression.

\author{
Романова Елена Юрьевна \\ Дочент, Российский государственный социальный \\ университет, г. Москва \\ romanova.elena.yu@yandex.ru
}

Аннотация. Системный подход к изучению проблемы подразумевает системный характер самого подхода. В данной работе рассматриваются варианты построения классификатора для распознавания депрессивных состояний членов общества методами машинного обучения. Предлагается методика построения классификатора на основе данных мониторинга физической активности и характеристик образа жизни.

Ключевые слова: анализ информации, системный подход, классификация, депрессия. истемный подход к изучению проблемы подразумевает системный характер самого подхода. Адекватность описания проблемы определяется полнотой инструментов исследования. В данной работе рассматриваются варианты построения классификатора для распознавания депрессивных состояний членов общества методами машинного обучения.

Описание проблемы. Депрессивные расстройства являются одними из самых распространенных проблем со здоровьем, в процессе исполнения трудовых функций коллективом они вызывают функциональные нарушения и снижают производительность сотрудников в большей степени, чем любые другие заболевания

Депрессивные расстройства влияют на социально-экономическую сторону жизни как на индивидуальном, так и на организационном уровнях. В исследовании [1] получены оценки потерь продуктивного времени и связанные с этим затраты среди работников с большим депрессивным расстройством по сравнению с контрольной группой: из-за прогулов и снижения производительности во время присутствия на работе рабочие с депрессивными расстройствами теряли 33,4\% от их средней годовой заработной платы, тогда как в контрольной группе средние затраты составили 2,5\% от годовой заработной платы.

В работе [2] оценивались потери производительности из-за прогулов и присутствия на работе с депрессией и их детерминанты у пациентов из пяти городов
Колумбии. Вероятность отсутствия на работе была на 17 процентных пунктов ниже у пациентов с хорошей самооценкой психического здоровья по сравнению с теми, кто оценивал его плохо, что подтверждает высокое экономическое бремя депрессии.

Как отмечено в [3], Политической декларацией $\mathrm{OOH}$ 2019 г было признано, что содействие психическому благополучию и профилактика состояний психического здоровья способствуют сокращению на одну треть преждевременной смертности от неинфекционных заболеваний. Плохое психическое здоровье пагубно сказывается на когнитивных, поведенческих, эмоциональных, социальных и взаимоотношениях человека. Способность участвовать в работе снижается из-за снижения производительности, снижения уровня безопасности труда или трудностей с сохранением или получением работы.

По оценкам, общие психические расстройства, такие как депрессия и тревожные расстройства, обходятся мировой экономике - окружающей среде в 1 триллион долларов США ежегодно.

По данным Всемирной организации здравоохранения во всем мире психическими и поведенческими расстройствами страдают около 460 миллионов человек, т.е. в среднем 6\% населения мира. Для России выявлена высокая степень корреляции динамики психологического состояния населения и смертности за последние десятилетия. Системный подход к исследованию демографических процессов стребует учета не только мате- 
риальных, но и психогенных детерминант народонаселения $[11,12]$.

Проблема этиологии, распространенности, патогенеза, диагностики, лечения и профилактики депрессий выходит за рамки психиатрической науки. До двух из десяти пациентов, которые обращаются за консультацией, лечением или диагностикой к врачу общей практики, семейному врачу, врачу-терапевту, находится в состоянии депрессивного расстройства. Каждый шестой случай в клинической практике приводит к инвалидизации пациента. Очень незначительный процент заболевших жалуется именно на психические или поведенческие проблемы. В связи с чем в клинической практике затруднительно отделить психическую или поведенческую патологию от соматических заболеваний $[5,6,7,8,9]$.

Baрианты решения проблемы. Методы ранней диагностики и профилактики депрессий изучены недостаточно. Традиционные способы оценки психоэмоционального состояния (психологические опросники) в определенной степени субъективны, поэтому требуются более объективные методы.

В исследовании [10] получены статистически значимые корреляции мощности и коэффициента межполушарной когерентности основных ритмов ЭЭГ с уровнем тревоги и депрессии.

Другой подход связан с анализом повседневного языка, тестов, например, постов в социальных сетях. Считается, что повседневный язык содержит множество лингвистических маркеров, способных сигнализировать о состоянии человека, написавшего текст.

В работе [13] авторы обучили нейросеть для распознавания депрессии по произвольной речи человека без контекста, при этом точность классификации составила $71 \%$, а полнота выявления болезни - $83 \%$ от всех больных в выборке.

В работе [14] удалось идентифицировать некоторые характеристики речи, указывающие на скрытую депрессию и тревожность у детей: монотонная речь, постоянное повторение одних и тех же рассказов.

Распознавание эмоций используется в различных реальных приложениях, где эмоциональное состояние человека служит признаком успешной работы этих систем, например, системы оценки качества обслуживания, рекомендательные системы заинтересованы в обнаружении эмоционального состояния человека. Системная архитектура emHealth [16] представляет собой интеллектуальную систему рекомендаций по здоровью с прогнозированием депрессии.
В [17] разработана модель на основе методов машинного обучения для измерения степени выраженности симптомов депрессии на основе объединения распознавания речи, компьютерного зрения и NLP (обработки естественного языка). Модель демонстрирует чувствительность 83,3\% и специфичность 82,6\%.

В [18] построена модель выявления депрессии по текстам в Facebook, метрика AUC = 0,69, выявлены следующие предикторы депрессии: эмоциональные (грусть), межличностные (одиночество, враждебность) и когнитивные (озабоченность собой, размышления). Такой метод может помочь находить людей, которым требуется психологическая поддержка и постановка диагноза. Ненавязчивая оценка депрессии с помощью социальных сетей у согласившихся людей может стать возможным в качестве масштабируемого дополнения к существующим процедурам скрининга и мониторинга [22].

В работе [19] исследователи разработали методологию, которая объединяет несколько алгоритмов машинного обучения для распознавания депрессии по тому, как звучит его голос.

Исследователями [20] разработана нейросеть, определяющая уровень депрессии пациента по его записи в видео- или аудиоформате. Полнота распознавания $77 \%$.

В [21] построены классификаторы депрессии у пользователей Вконтакте по различным параметрам активности и психолингвистическим маркерам их текстов.

В настоящей работе предлагается методика построения еще одного варианта классификатора по данным мониторинга физической активности и показателям, которые можно отнести к характеристикам образа жизни - объективно наблюдаемым величинам. Показатели физической активности поступают с носимых устройств, которые сейчас довольно популярны. Характеристика образа жизни в данном исследовании ограничивалась списком употребляемых продуктов питания и данными об участии в трудовой деятельности.

Исходные данные для исследования взяты из 20052006 National Health and Nutrition Examination Survey (NHANES) [23]. Данные о физической активности представлены в виде временных рядов, данные о характеристиках образа жизни - в виде ответов респондентов на вопросы.

Изложим методику формирования обучающих данных. Целевым значением считался ответ на вопросы с идентификатором DPQ020 для определения наличия депрессии о том, как часто чувствовал ли себя ре- 


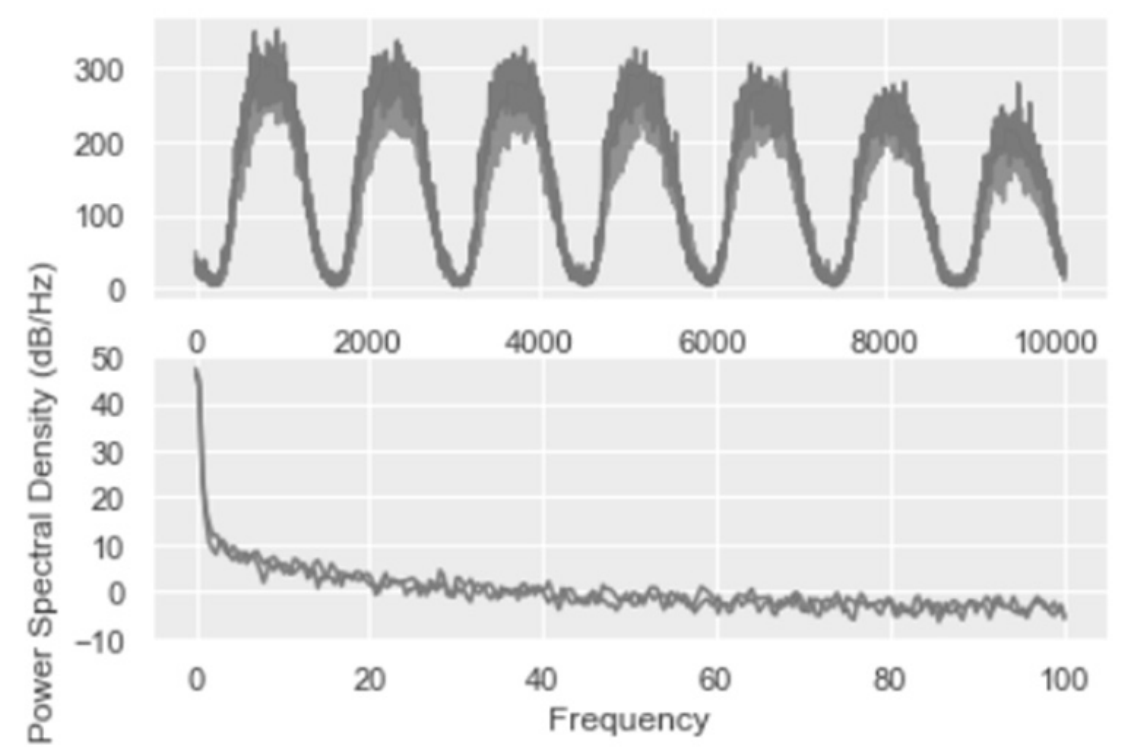

Рис. 1. Графики усредненных физических активностей людей с наличием и отсутствием депрессии (вверху), спектральные плотности мощности

спондент за последние 2 недели в подавленном или депрессивном состоянии. Положительные ответ соответствовал признаку наличия депрессии, отрицательный - отсутствию. Ответы были получены от 4831 человек, из которых положительными оказались 1062 ответа.

Объем данных о физической активности, снимавшихся ежеминутно в течение недели не позволяет строить математические модели машинного обучения непосредственно на этих данных на РС в условиях отсутствия мощных ресурсов (например, при RAM меньше 32 Гб).

Поэтому для извлечения существенных признаков из данных о физической активности применялись два метода. Заметим, что была необходимость в предварительной обработке данных, т.к., по сообщениям В ряде источников [24,25] при возрастании нагрузки (интенсивный бег) показания применяемого акселерометра Actigraph (полное название the Manufacturing Technology Incorporated Actigraph and the Computer Science Applications) выше 10000 перестают отражать степень реальной нагрузки, и соответствующие выбросы были заменены пороговым значением.

Первый метод - применение скрытой марковской модели и последующий метод главных компонент (РСА), был успешно применен для оценки биологического возраста в работе [26] и последующих разработках коллектива авторов [26]. На рисунке 1 приведены графики усредненных физических активностей людей с наличием и отсутствием депрессии, а также спектральные плотности мощности показателей. При усреднении соблюдалась пропорция возрастных категорий, а само усреднение возможно в силу принадлежности респондентов к одной системе - нахождению в одной стране.

Проведенный при кросс-валидации анализ указывает на статистическую значимость различий.

Для построения матрицы переходов скрытой гауссовой марковской модели (НММ) требовалось задать число скрытых состояний. Оно было определено экспериментально как точка равновесия между увеличивающимся временем расчета модели и уменьшающейся погрешностью модели. Приемлемые результаты были достигнуты при числе состояний 7 (рисунок 2, справа). К полученной матрице переходов применялся метод PCA, и семь первых компонент брались как признаки для обучения модели.

Второй метод - к данным о физической активности применялась небольшая нейронная сеть с типом архитектуры энкодер-декодер, посредством которой количество признаков физической активности снизилось до 578 (выход с последнего слоя энкодера). К ним также был применен РСА.

Данные опросов являлись небинарными категориальными переменными, и к ним была применена стандартная для машинного обучения предобработка. Наиболее существенные вопросы будут приведены ниже.

Для построения классификаторов применялись методы градиентного бустинга, случайного леса и логистическая регрессия. Классификатор на основе градиентного бустинга, построенный только на признаках о физиче- 
1.0

0

20 40 Возраст

1.0

0 20 40 Bозраст

\section{0}

0 20 40 Возраст
1.0

0

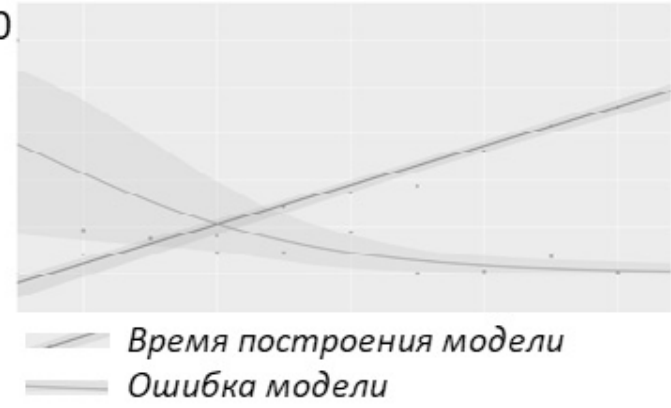

1.0

0

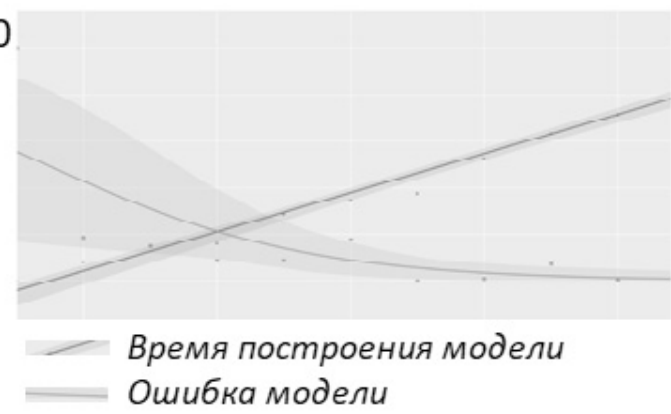

1.0

0

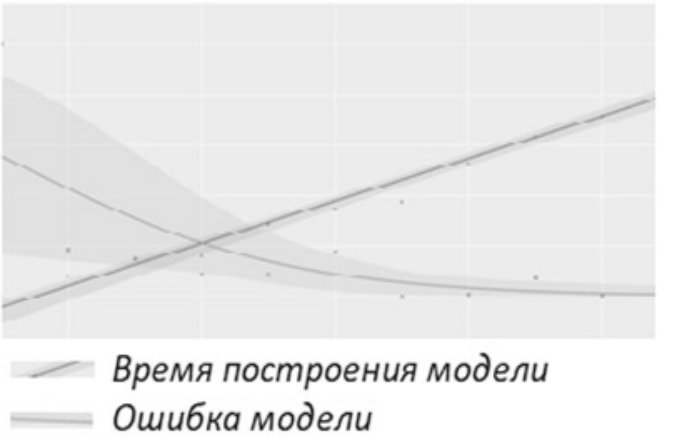

Рис. 2. Регрессия первых и вторых компонент РСА на возраст (слева), времени построения и ошибки модели НММ на число состояний (справа)

ской активности, имел значения метрик precision 0.82 и recall 0.76, что является хорошим результатом, на уровне методов, рассмотренных выше в обзоре. Классификатор, построенный на матрице признаков на компонентах РСА и характеристиках, связанных с образом жизни, увеличило метрики до средних значений: precision 0.90 и recall 0.82. При этом интересно отметить, что первые две компоненты РСА, примененного к признакам, полученным от нейросети, имеют коэффициенты детерминации 0.81 и 0.66 с возрастом от 18 до 40 и более 40 лет соответственно (рисунок 2).

Аналогичную корреляцию с возрастом более 40 лет на уровне 0.60 выявили авторы работы [27] относительно первой компоненты РСА матрицы переходов. Выявленный факт дает возможность для построения более точных моделей при учете демографических признаков, чего в данном исследовании намеренно не делалось.

В результате данного исследования были выявлены признаки, входившие по крайней мере дважды в топ-30 по важности для трех классификаторов. В эти признаки вошли все 6 первых компонент РСА матрицы переходов и 7 первых компонент матрицы выходов энкодера нейронной сети. Проведенный анализ с помощью критерия хи-квадрат Пирсона для качественных данных подтвердил существенность различий (на уровне значимости 5\%, $p$-value $<0.05)$ по этим признакам для групп респондентов (таблица 1).

На рисунке 3 наглядно представлены различия в ответах на некоторые вопросы. Наличие депрессивных 
Таблица 1. Значимые для классификации вопросы (американская аудитория)

\begin{tabular}{|c|c|c|}
\hline $\begin{array}{l}\text { Идентификатор вопроса } \\
\text { в NHANES }\end{array}$ & Текст вопроса для двух групп респондентов & p-value \\
\hline PAD200 & Активная активность за последние 30 дней & $9.7373 e-10$ \\
\hline PAD320 & Умеренная активность за последние 30 дней & $4.9742 \mathrm{e}-09$ \\
\hline PAQ180 & Средний уровень физической активности каждый день & $1.4737 \mathrm{e}-10$ \\
\hline FFQ0103 & Вы едите чипсы? & $2.6938 \mathrm{e}-12$ \\
\hline OCD150 & Тип работы, проделанной на прошлой неделе & $2.1739 \mathrm{e}-16$ \\
\hline FFQ0006 & Как часто употребляете другие морсы? & $3.6082 \mathrm{e}-14$ \\
\hline PAQ100 & Задачи по дому / двору за последние 30 дней & $9.8053 e-06$ \\
\hline FFQ0137 & Вы едите сливочный сыр? & $1.1234 \mathrm{e}-07$ \\
\hline FFQ0139 & Вы едите продукты с маслами? & 0.0001 \\
\hline FFQ0001 & Как часто пьете томатный сок? & 0.0171 \\
\hline FFQ0004 & Как часто пьете виноградный сок? & 0.0011 \\
\hline FFQ0007 & Как часто пьете молоко как напиток? & 0.0001 \\
\hline FFQ0012 & Вы пьете спиртное? & $2.1656 \mathrm{e}-07$ \\
\hline FFQ0016 & Вы едите яблоки? & $1.5375 e-13$ \\
\hline FFQ0017 & Вы едите груши? & $2.9716 \mathrm{e}-09$ \\
\hline FFQ0020 & Вы едите сухофрукты? & $2.4685 e-06$ \\
\hline FFQ0021 & Вы едите персики? & $1.5457 \mathrm{e}-05$ \\
\hline FFQ0024 & Съедите свежую клубнику? & 0.0006 \\
\hline FFQ0029 & Вы едите сырую зелень? & 0.0032 \\
\hline FFQ0030 & Вы едите салат из капусты? & $2.0331 \mathrm{e}-06$ \\
\hline FFQ0032 & Вы едите морковь? & $1.7057 \mathrm{e}-07$ \\
\hline FFQ0051 & Вы едите кетчуп? & $1.0905 \mathrm{e}-14$ \\
\hline FFQ0054 & Вы едите чили? & $8.5256 \mathrm{e}-12$ \\
\hline FFQ0088 & Вы едите ливер? & 0.0010 \\
\hline FFQ0121 & Вы едите другие конфеты? & $1.6560 \mathrm{e}-07$ \\
\hline FFQ0133 & Вы едите масло на хлебе? & 0.0024 \\
\hline FFQ0136 & Вы едите майонез? & $1.6227 \mathrm{e}-09$ \\
\hline FFQ0035 (для сравнения) & Вы едите кукурузу? & 0.8953 \\
\hline
\end{tabular}

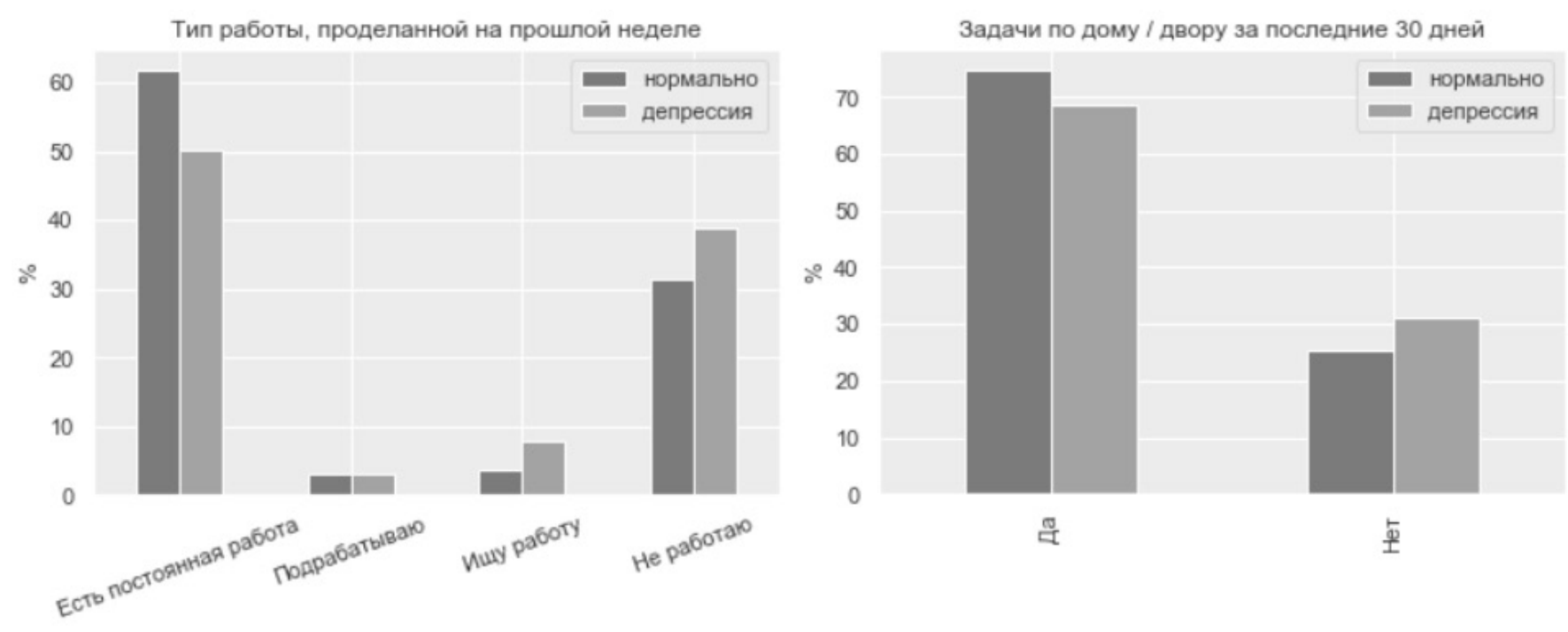

Рис. 3. Различия в ответах групп респондентов 
состояний обозначено как «депрессия», отсутствие как «нормально» исключительно в иллюстративных целях.

Таким образом, в результате данного исследования можно сделать вывод, что наиболее значимыми признаками наличия депрессии респондентов без учета демографических данных можно назвать следующие: показатели физической активности; трудовая активность; социальная вовлеченность. Возможно построение классификатора только на основе показаний акселерометра, но лучший результат классификации на представленном наборе данных получается при их комбинации с характеристиками образа жизни.

Критериями отбора вариантов классификаторов являются метрики качества классификации: доля правильных ответов, точность, полнота, F1-мера и др. Больший вес в связи с характером решаемой проблемы имеет полнота, т.е.доля страдающих депрессией, правильно определенная ансамблем классификаторов.

\section{Значимость решения проблемы}

Мировым сообществом предпринимаются действия по построению классификаторов на основании системного подхода, использующего всё многообразие форм проявлений личности. В случае построения оптимального комплексного решения реализация его принесет существенную выгоду.

Так, в [4] предложено инвестиционное обоснование для расширения охвата эффективным лечением депрессии и тревожных расстройств. Показано, что при умеренном улучшении на 5\% как трудоспособности, так и производительности труда в результате лечения, соотношение получаемых выгод к затратам составляет 2.3-3 к 1, если учитываются только экономические выгоды, и 3.3-5.7 к 1, когда также включается значение прибыли для здоровья.

\section{ЛИТЕРАТУРА}

1. Jong-Min Woo, Won Kim, Tae-Yeon Hwang, etc. Impact of Depression on Work Productivity and Its Improvement after Outpatient Treatment with Antidepressants // Value in Health.— 2011.— № 4. V. 14.—P. 475-482, https://doi.org/10.1016/j.jval.2010.11.006.

2. Uribe José Miguel, Pinto Masis, Diana \& Vecino Ortiz, etc. Presenteeism, Absenteeism, and Lost Work Productivity among Depressive Patients from Five Cities of Colombia. //Value in Health Regional Issues.— 2017.—V. 14.— P. 15-19. https://doi.org/10.1016/j.vhri.2017.03.001.

3. Сайт Всемирной организации здравоохранения https://www.who.int/mental_health/prevention/guidelines_mental_health_work

4. Cuijpers P., Chisholm Dr., Sweeny K. etc. Scaling-up treatment of depression and anxiety: A global return on investment analysis. // The Lancet Psychiatry. 2016. - V. 3. https://doi.org/ 10.1016/S2215-0366(16)30024-4.

5. Nogueira M.H., Yasuda C. L., Coan A. C., Kanner A. M., Cendes F. Concurrent mood and anxiety disorders are associated with pharmacoresistant seizures in patients with MTLE. // Epilepsia.— 2017.— № 58. V. 7.—P. 1268-1276. https://doi.org/10.1111/epi.13781.

6. Collaborators GBDN. Global, regional, and national burden of neurological disorders, 1990-2016: a systematic analysis for the Global Burden of Disease Study 2016. // Lancet Neurol._ 2019. № 5. V. 18. — P.:459-80. https://doi.org/10.1016/S1474-4422(18)30499-X.

7. Krishnan V. Depression and Anxiety in the Epilepsies: from Bench to Bedside. // Curr Neurol Neurosci — 2020.— № 20. V. https://doi.org/10.1007/s11910-020 01065-z

8. Александер Ф. Психосоматическая медицина. Принципы и практическое применение / Ф. Александер: пер. с англ.— М.: ЭКСМ0-Пресс, 2002. — 352 с.

9. Александровский Ю. А. Пограничные психические расстройства при соматических заболеваниях / Ю. А. Александровский // Психиатрия и психофармакотерапия.— 2002.— № 1.——C. 4-7.

10. Газенкамп $ф$ К.А., Дмитренко Д. В., Карнаухов В. Е., Фирсова Д. А. Корреляция мощности основных ритмов электроэнцефалограммы и коэффициента когерентности с уровнем тревоги и депрессии в юношеском возрасте // Доктор.Ру.— 2019. — № 6. V. 161.—C. 53-57. https://doi.org/10.31550/1727-23782019-161-6-53-57

11. И.А. Гундаров. Общественное сознание как предмет системного анализа причин демографических процессов // Труды ИСА РАН. — 2016. — № 2. Т. 66.

12. Винокур В.А., Новикова Ирина Альбертовна Системный анализ процесса интеграции в психотерапии // Экология человека. — 2015.—№ 4.

13. Nawshad Farruque et al., Augmenting Semantic Representation of Depressive Language: From Forums to Microblogs // Machine Learning and Knowledge Discovery in Databases. — 2020. https://doi.org/10.1007/978-3-030-46133-1_22

14. E.W. McGinnis et al. Giving Voice to Vulnerable Children: Machine Learning Analysis of Speech Detects Anxiety and Depression in Early Childhood // IEEE Journal of Biomedical and Health Informatics, — 2019. — № 6. V. 23. — P. 2294-2301, https://doi.org/10.1109/JBHI.2019.2913590.

15. Chiu I., Piguet 0., Diehl-Schmid J., RiedI L., Beck J., Leyhe T., Holsboer-TrachslerE., Kressig R. W., Berres M., Monsch A. U., et al. Facial Emotion Recognition Performance Differentiates Between Behavioral Variant Frontotemporal Dementia and Major Depressive Disorder. J. Clin. Psychiatry.— 2018. — № 79. https://doi.org/10.4088/ JCP. $16 \mathrm{~m} 11342$.

16. Yang S., Zhou P., Duan K., Hossain M. S., Alhamid M. F. emHealth: Towards emotion health through depression prediction and intelligent health recommender system. Mob. Netw. Appl.— 2017.— № 23.—P. 216-226. https://doi.org/10.1007/s11036-017-0929-3

17. A. Haque, M. Guo, A. S. Miner, L. Fei-Fei Measuring depression symptom severity from spoken language and 3D facial expressions // arXiv preprint arXiv:1811.08592, 2018 - arxiv.org 
18. Johannes C. Eichstaedt, Robert J. Smith, Raina M. Merchant etc. Facebook language predicts depression in medical records // Proceedings of the National Academy of Sciences — 2018. — № 44. V. 115.— P. 11203-11208. https://doi.org/10.1073/pnas. 1802331115

19. Mashrura Tasnim, Eleni Stroulia. Detecting Depression from Voice. // Canadian Conference on Artificial Intelligence. — 2019. https://doi.org/10.1007/978-3-03018305-9_47

20. Rob Matheson. Model can more naturally detect depression in conversations. Neural network learns speech patterns that predict depression in clinical interviews. // MIT News Office August 29, 2018.

21. Н.В. Кисельникова, М. А. Станкевич, М. М. Данина, Е. А. Куминская, Е. В. Лаврова. Выявление информативных параметров поведения пользователей социальной сети Вконтакте как признаков депрессии // Психология. Журнал Высшей школы экономики.— 2020.— № 1. T. 17.— C. 73-88. https://doi. org/10.17323/1813-8918-2020-1-73-88

22. Marcel Trotzek, Sven Koitka, Christoph M. Friedrich Utilizing Neural Networks and Linguistic Metadata for Early Detection of Depression Indications in Text Sequences // April 2018 IEEE Transactions on Knowledge and Data Engineering 32(3):588-601, https://doi.org/10.1109/TKDE.2018.2885515

23. Сайт National Center for Health Statistics https://wwwn.cdc.gov/nchs/nhanes/continuousnhanes/

24. Ellis K., Kerr J., Godbole S., Staudenmayer J., Lanckriet G. Hip and Wrist Accelerometer Algorithms for Free-Living Behavior Classification. // Medicine and Science in Sports and Exercise.— 2016. — № 48. V. 5.—P. 933-940. https://doi.org/10.1249/mss.00000000000000840.

25. Crouter S.E., Clowers K. G., \& Bassett D. R., Jr (2006). A novel method for using accelerometer data to predict energy expenditure. // Journal of applied physiology.1985.—№ 100. V. 4. — P. 1324-1331. https://doi.org/10.1152/japplphysiol.00818.2005

26. Pyrkov Timothy, Getmantsev Evgeny, Zhurov Boris, etc. Quantitative characterization of biological age and frailty based on locomotor activity records. // — 2018. № 10. https://doi.org/10. 2018. doi:10.18632/aging.101603.

(с) Романова Елена Юрьевна ( romanova.elena.yu@yandex.ru ).

Журнал «Современная наука: актуальные проблемы теории и практики»

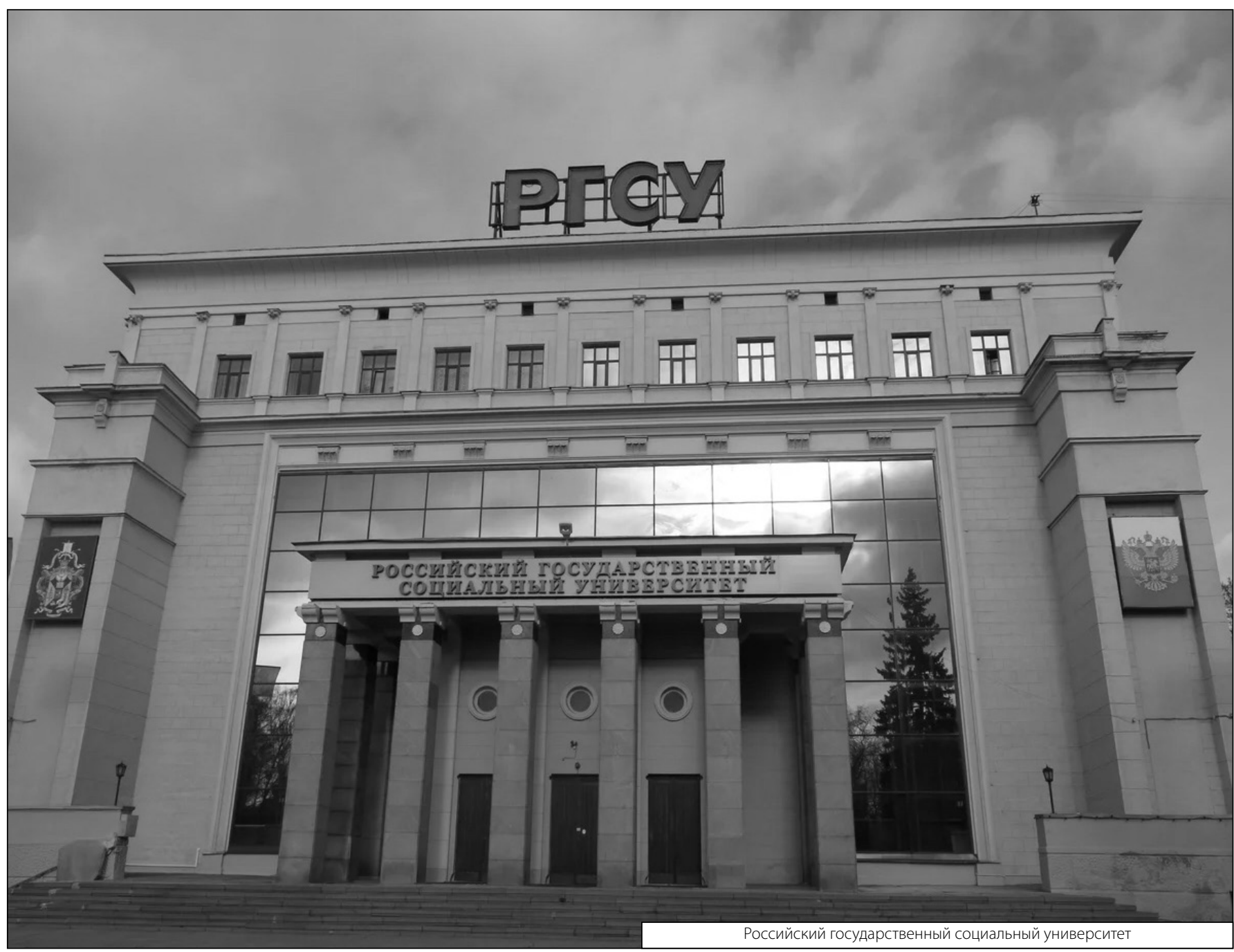

